\title{
Fortbildungspflicht
}

\section{Die nächste Punkterunde hat längst begonnen}

\author{
Vertragsärzte, die nicht alle fünf Jahre ihre 250 CME-Punkte \\ nachweisen, müssen tatsächlich mit Honorarkürzungen rechnen. \\ In der Vergangenheit hat das zwar nur wenige Ärzte getroffen. Aber \\ die KVen scheinen ernst zu machen.
}

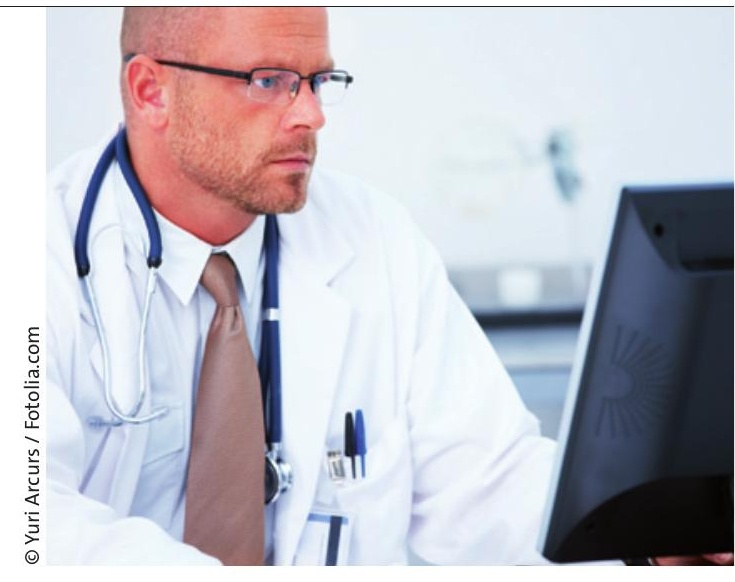

Alle fünf Jahre müssen Vertragsärzte ihre kontinuierliche Fortbildung nachweisen. Andernfalls droht Honorarverlust.
$\mathrm{D}$ ie meisten Vertragsärzte kommen ihrer Fortbildungspflicht nach. Das hat sich im Sommer vergangenen Jahres gezeigt, als die Ärzte erstmals belegen mussten, dass sie innerhalb von fünf Jahren 250 CME-Punkte gesammelt haben. Damals warteten die Kassenärztlichen Vereinigungen (KVen) mit Quoten von 95-98\% ihrer Mitglieder auf, die das Ziel erreicht hatten.

Doch was vielen Ärzten nicht präsent ist: Die Uhr tickt seitdem unerbittlich weiter. Die niedergelassenen Ärzte, die im Juli 2009 nachgewiesen haben, dass sie ihre Fortbildungspflicht erfüllt haben, müssen dies im Juli 2014 wieder tun - und immer wieder, alle fünf Jahre. Und in der Zwischenzeit müssen die Ärzte, die sich seitdem niedergelassen haben, jeweils fünf Jahre nach ihrer Niederlassung zum Quartalsende, ihrer Fortbildungspflicht nachgekommen sein. Die KVen fordern daher jedes Quartal die aktuellen Zahlen von den Kammern ein, weil der Fünfjahreszeitraum dann von Neuem beginnt und natürlich auch für später zugelassene Ärzte gilt. Rechtsgrundlage für diese Fortbildungspflicht ist $\$ 95 \mathrm{~d}$ Absatz 1 SGB V.

\section{Bei fehlendem Nachweis drohen Sanktionen}

Mittlerweile ist deutlich geworden, dass die KVen Sanktionen, die fällig werden, weil Ärzte ihrer Fortbildungspflicht nicht nachkommen, tatsächlich aussprechen. Für die ersten vier Quartale, die auf den Fünfjahreszeitraum folgen, muss das Honorar dem Gesetz zufolge um 10\% und ab dem darauf folgenden Quartal um $25 \%$ gekürzt werden.
„Diese Honorarkürzungen können Ärzte vermeiden, indem sie die benötigte Fortbildung so schnell wie möglich nachholen - und zwar innerhalb von zwei Jahren ganz oder zumindest teilweise“, erklärt Kirsten Warweg von der KV Bayerns. Warweg: „Die Honorarkürzung endet nach Ablauf des Quartals, in dem der vollständige Fortbildungsnachweis erbracht wird.“

Das haben seit Juli 2009 auch viele der Betroffenen getan, wie die aktuellen Zahlen aus den KVen zeigen. In Hessen hätten 54\% der Ärzte und Psychotherapeuten, die die erste Frist versäumt hatten, die fehlenden Punkte nachgereicht. Wird der Nachweis nicht erbracht, droht ab dem neunten Quartal der Zulassungsentzug.

\section{Fortbildungseifer lässt tendenziell nach}

Gerade neu niedergelassene Ärzte sollten die drohenden Sanktionen durchaus ernst nehmen. Denn wer sich bei den KVen umhört, erfährt schnell, dass die Werte nach den hervorragenden Zahlen beim ersten Stichtag in einigen KVen etwas abbröckeln. Das dürfte vor allem daran liegen, dass das Thema zum einen bei vielen Ärzten direkt nach der Niederlassung nicht so präsent ist, weil andere Sorgen im Vordergrund stehen. Zum anderen haben in den vergangenen Jahren KVen, Kammern und auch die Fachpresse ständig über das Thema berichtet. Jetzt, wo es um viel weniger Ärzte geht, hat die Aufmerksamkeit etwas nachgelassen.

Die Folge: In Bayern zum Beispiel lag der Anteil der Vertragsärzte, die ihre Frist versäumt haben, bei stolzen $48 \%$ im ersten
Quartal 2010 und bei 37\% im zweiten Quartal 2010. Es ging allerdings nur um wenige Ärzte: In I/2010 versäumten 44 Ärzte und Psychotherapeuten ihre Fortbildungsfrist. Auch in anderen KVen sind die Quoten säumiger Mitglieder gestiegen, jedoch nicht so deutlich wie in Bayern, und auch nicht überall.

In Sachsen-Anhalt etwa mussten in den Quartalen nach II/2009 nur 11, 18, 28 und in II/2010 schließlich 34 Ärzte und Psychotherapeuten ihren Forbildungsnachweis erbringen. In den vier Quartalen gab es nur zwei Ärzte, die ihre Pflicht schuldig blieben. Insgesamt musste die KV lediglich 38 Ärzten das Honorar kürzen.

Im weit größeren KV-Bereich Westfalen-Lippe wurde bisher das Honorar von 207 Ärzten und Psychotherapeuten gekürzt, weil die nötigen CME-Punkte fehlten. Dabei haben die Vertragsärzte in III/2009 zu 98,7\%, in IV/2009 zu 92,4\%, in $\mathrm{I} / 2010 \mathrm{zu} 95,2 \%$ und in II/2010 zu 97,7\% ihre Fortbildungsverpflichtung eingehalten.

Die steigenden Werte in manchen KVen liegen auch nicht an einer höheren Messlatte. 250 CME-Punkte müssen Ärzte und Psychotherapeuten weiterhin alle fünf Jahre nachweisen. Und dass dieses Ziel gut erreichbar ist, zeigen zum Beispiel die Werte aus Hamburg: Im vergangenen Jahr meldete die KV der Hansestadt, dass 97,3\% ihrer Mitglieder die Fortbildungspflicht erfüllt hätten - mit einer durchschnittlichen Punktezahl von 474 Punkten.

\section{Rebekka Höhl, Hauke Gerlof}

Neu Isenburg 